\title{
Zur Zulässigkeit der Umsetzung der EG-Blutrichtlinien 2002/98/EG und 2004/33/EG in nationales Recht durch Richtlinien der Bundesärztekammer nach §§ 12 und 18 Transfusionsgesetz*
}

\author{
Horst Hasskarl \\ Ludwigshafen, Deutschland
}

\section{Ausgangssituation}

Die Richtlinie 2002/98/EG des Europäischen Parlaments und des Rates vom 27. Januar 2003 zur Festlegung von Qualitätsund Sicherheitsstandards für die Gewinnung, Testung, Verarbeitung, Lagerung und Verteilung von menschlichem Blut und Blutbestandteilen und zur Änderung der Richtlinie 2001/83/EG [ABl. EU Nr. L 33/30 vom 8. Februar 2003] ist gemäß Artikel 32 Absatz 1 bis zum 8. Februar 2005 durch die Mitgliedsstaaten in nationales Recht umzusetzen. Hierzu müssen die Mitgliedsstaaten die Rechts- und Verwaltungsvorschriften in Kraft setzen, die erforderlich sind, um dieser Richtlinie nachzukommen. Gleichfalls bis zu diesem Zeitpunkt umzusetzen ist die Richtlinie 2004/33/EG der Kommission vom 22. März 2004 zur Durchführung der Richtlinie 2002/98/EG des Europäischen Parlaments und des Rates hinsichtlich bestimmter technischer Anforderungen für Blut und Blutbestandteile [ABl. EU Nr. L 91/25 vom 30. März 2004]. In Deutschland ist die Umsetzung dieser Richtlinien nur teilweise durch den Entwurf eines Ersten Gesetzes zur Änderung des Transfusionsgesetzes und arzneimittelrechtlicher Vorschriften geplant [Bundestag-Drucksache 15/3593 vom 14. Juli 2004]. Dieser Entwurf wurde am 10. November 2004 vom Ausschuss für Gesundheit und soziale Sicherung in leicht veränderter Form angenommen [BT-Drs. 15/4174 vom 10. November 2004]. Diese Änderungen beziehen sich im Wesentlichen auf die Freiwilligkeit und die Unentgeltlichkeit von Blut- und Plasmaspenden und berühren die hier zu untersuchende Problematik nicht.

Gemäß $§ 12$ Absatz 1 und $\S 18$ Absatz 1 des Gesetzes zur Regelung des Transfusionswesens (Transfusionsgesetz; TFG)

\footnotetext{
* unter Mitwirkung von Rechtsassessor Jens Kuchta, Mannheim,
} Deutschland vom 1. Juli 1998 [BGBl. I S. 1752], zuletzt geändert durch die Achte Zuständigkeitsanpassungsverordnung vom 25. November 2003 [BGBl. I S. 2304] stellt die Bundesärztekammer den allgemein anerkannten Stand der medizinischen Wissenschaft und Technik zur Gewinnung von Blut und Blutbestandteilen ( 12 Absatz 1 TFG) bzw. zur Anwendung von Blutprodukten (§ 18 Absatz 1 TFG) in Richtlinien im Einvernehmen mit dem Paul-Ehrlich-Institut fest. In diese so genannten Hämotherapierichtlinien (Richtlinien zur Gewinnung von Blut- und Blutbestandteilen und zur Anwendung von Blutprodukten (Hämotherapie) vom 7. Juli 2000 [Bundesgesundheitsblatt 2000, S. 555 ff.; 2001, S. 1240 f.; 2004, S. 207] sollen diejenigen von den EG-Richtlinien vorgeschriebenen Inhalte eingearbeitet werden, die nicht durch eine Änderung des TFG selbst umgesetzt werden [BT-Drs. 15/3593 vom 14. Juni 2004, S. 12]. Damit soll die Umsetzung von EG-Richtlinien zu einem erheblichen Teil durch das Instrument der Richtlinien der Bundesärztekammer erfolgen. Außerdem ist nunmehr eine Bekanntmachung der Hämotherapierichtlinien im Bundesanzeiger und nicht mehr im Bundesgesundheitsblatt vorgesehen. Die insoweit maßgeblichen Normen des TFG lauten (die durch den Gesetzentwurf beabsichtigten Änderungen sind kursiv gesetzt) wie folgt:

$\$ 12$

Stand der medizinischen Wissenschaft und Technik zur Gewinnung von Blut und Blutbestandteilen

(1) Die Bundesärztekammer stellt im Einvernehmen mit der zuständigen Bundesoberbehörde und nach Anhörung von Sachverständigen unter Berücksichtigung der Richtlinien und Empfehlungen der Europäischen Union, des Europarates und der Weltgesundheitsorganisation zu Blut und Blutbestandteilen in Richtlinien den allgemein anerkannten Stand der medizinischen Wissenschaft und Technik für

1. die Sachkenntnis des Personals der Spendeeinrichtung,

2. die Auswahl der spendenden Personen und die Durchführung der Auswahl,

3. die Identifizierung und Testung der spendenden Personen,

\begin{tabular}{ll}
\hline KARGER & $\oplus$ c 2005 S. Karger GmbH, Freiburg \\
Fax +497614520714 & Accessible online at: \\
$\begin{array}{l}\text { E-mail Information@Karger.de } \\
\text { www.karger.com }\end{array}$ & www.karger.com/tmh
\end{tabular}

\section{KARGER}

www.karger.com
Dr. Horst Hasskarl

Bleichstraße 57

67061 Ludwigshafen, Deutschland

E-mail hasskarl-law@t-online.de 
4. die durchzuführenden Laboruntersuchungen,

5. die ordnungsgemäße Entnahme der Spenden,

6. die Eigenblutentnahme,

7. die Gewinnung von Plasma für die Herstellung spezieller Immunglobuline, insbesondere die Spenderimmunisierung,

8. die Separation von Blutstammzellen und anderen Blutbestandteilen, insbesondere die Vorbehandlung der spendenden Personen, und

9. die Dokumentation der Spendenentnahme

fest. Bei der Anhörung ist die angemessene Beteiligung von Sachverständigen der Fach- und Verkehrskreise, insbesondere der Träger der Spendeneinrichtung, der Plasmapoolprodukte herstellenden pharmazeutischen Unternehmer, der Spitzenverbände der Krankenkassen, der Deutschen Krankenhausgesellschaft sowie der zuständigen Behörden von Bund und Ländern sicherzustellen. Die Richtlinien werden von der zuständigen Bundesoberbehörde im Bundesanzeiger bekannt gemacht.

(2) Es wird vermutet, dass der allgemein anerkannte Stand der medizinischen Wissenschaft und Technik zu den Anforderungen nach diesem Abschnitt eingehalten worden ist, wenn und soweit die Richtlinien der Bundesärztekammer nach Absatz 1 beachtet worden sind.

$\$ 18$

Stand der medizinischen Wissenschaft und Technik zur Anwendung Blutprodukten

(1) Die Bundesärztekammer stellt im Einvernehmen mit der zuständigen Bundesoberbehörde und nach Anhörung von Sachverständigen unter Berücksichtigung der Richtlinien und Empfehlungen der Europäischen Union, des Europarates und der Weltgesundheitsorganisation zu Blut und Blutbestandteilen in Richtlinien den allgemein anerkannten Stand der medizinischen Wissenschaft und Technik für

1. die Anwendung von Blutprodukten einschließlich der Dokumentation der Indikation zur Transfusion, die Testung auf Infektionsmarker der zu behandelnden Personen anlässlich der Anwendung von Blutprodukten und die Anforderungen an die Rückstellproben,

2. die Qualitätssicherung der Anwendung von Blutprodukten in den Einrichtungen der Krankenversorgung und ihre Überwachung durch die Ärzteschaft,

3. die Qualifikation und die Aufgaben der im engen Zusammenhang mit der Anwendung von Blutprodukten tätigen Personen,

4. den Umgang mit nicht angewendeten Blutprodukten in den Einrichtungen der Krankenversorgung

fest. Bei der Anhörung ist die angemessene Beteiligung von Sachverständigen der Fach- und Verkehrskreise, insbesondere der Träger der Spendeneinrichtung, der Spitzenverbände der Krankenkassen, der Deutschen Krankenhausgesellschaft, der Kassenärztlichen Bundesvereinigung sowie der zuständigen Behörden von Bund und Ländern sicherzustellen. Die Richtlinien werden von der zuständigen Bundesoberbehörde im Bundesanzeiger bekannt gemacht.

(2) Es wird vermutet, dass der allgemein anerkannte Stand der medizinischen Wissenschaft und Technik zu den Anforderungen nach diesem Abschnitt eingehalten worden ist, wenn und soweit die Richtlinien der Bundesärztekammer nach Absatz 1 beachtet worden sind.

Zur Begründung für die Umsetzung der von den EG-Richtlinien vorgegebenen fachlichen Standards in den Hämotherapierichtlinien der Bundesärztekammer wird auf die Flexibilität der Hämotherapierichtlinien abgestellt [BundestagsDrucksache 15/3593, S. 12]: Wörtlich heißt es unter anderem:

Die Mindeststandards müssen in der nationalen Richtlinie der Bundesärztekammer und des Paul-Ehrlich-Instituts (Hämotherapierichtlinie) umgesetzt werden, die allerdings darüber hinaus gehende Standards fest- legen kann. Als entsprechend flexibles Instrument sind deshalb die Hämotherapierichtlinien der geeignete Ort zur Umsetzung der fachlichen Standards.

Die künftige Veröffentlichung der Hämotherapierichtlinien im Bundesanzeiger durch das Paul-Ehrlich-Institut als für Blutprodukte zuständige Bundesoberbehörde soll für einen amtlichen Charakter dieser Richtlinie sorgen [BT-Drs., a.a.O., S. 12]:

Um die Bedeutung der Hämotherapierichtlinien der Bundesärztekammer und des Paul-Ehrlich-Instituts stärker zu betonen, sollen die Richtlinien in Zukunft durch das Paul-Ehrlich-Institut (zuständige Bundesoberbehörde) im Bundesanzeiger bekannt gemacht werden. Es handelt sich um gesetzlich basierte Richtlinien mit hohem fachlichem, aber auch juristischem Gewicht. Die Bekanntmachung durch die Bundesoberbehörde in einem offiziellen Organ der Bundesregierung verleiht den Richtlinien einen amtlichen Charakter.

Im Folgenden soll überprüft werden, ob diese Art der Umsetzung mit den Vorgaben der Richtlinien 2002/98/EG und 2004/33/EG (vgl. Art. 32 Absatz 1 der Richtlinie 2002/98/EG und 9 Absatz 1 der Richtlinie 2004/33/EG) bzw. mit dem EGVertrag und der Rechtsprechung des EuGH zur Umsetzung von Richtlinien vereinbar ist. Darüber hinaus ist die Frage zu prüfen, ob die geplante Art der Umsetzung den deutschen verfassungsrechtlichen Vorgaben entspricht. Schließlich soll ein Vergleich zur Umsetzung von EG-Richtlinien im Bereich des Arzneimittelrechts gezogen werden.

\section{Vorgaben der EG-Blutrichtlinien}

Nach Artikel 32 Absatz 1 der Richtlinie 2002/98/EG und Artikel 9 Absatz 1 der Richtlinie 2004/33/EG sind die Mitgliedsstaaten gehalten, die zur Umsetzung der Richtlinie notwendigen Rechts- und Verwaltungsvorschriften bis 8. Februar 2005 in Kraft zu setzen. Dabei handelt es sich inhaltlich unter anderem um von der Blutspendeeinrichtung an die zuständige Behörde zu übermittelnde Informationen im Hinblick auf die Benennung, Zulassung, Anerkennung oder Erteilung einer Erlaubnis, um euroopaeinheitliche Definitionen, um die Informationen, die potentiellen Spendern zu erteilen bzw. von ihnen einzuholen sind einschließlich der Einzelheiten der Einwilligungserklärung des Spenders, um Eignungskriterien für die Spender (Zulassungskriterien und Ausschlusskriterien), um Bedingungen für Lagerung, Transport und Verteilung und um Qualitäts- und Sicherheitsanforderungen an Blut und Blutbestandteile. Es ist daher zunächst zu untersuchen, ob die in Deutschland geplante Form der Umsetzung mit dem Erlass von Rechts- bzw. Verwaltungsvorschriften einhergeht. Maßgeblich für diese Frage ist zunächst die Rechtsform der Bundesärztekammer, die für die Umsetzung der Richtlinie sorgen soll. Im Anschluss daran sind die von der Bundesärztekammer erlassenen Richtlinien rechtlich zu qualifizieren. 
kel 32 Absatz 1 der Richtlinie 2002/98/EG bzw. 9 Absatz 1

Die Bundesärztekammer (Arbeitsgemeinschaft der Deutschen Ärztekammern) ist die Spitzenorganisation der ärztlichen Selbstverwaltung. Sie vertritt die berufspolitischen Interessen der 388201 Ärztinnen und Ärzte (Stand: 31. Dezember 2003) in der Bundesrepublik Deutschland. Die Bundesärztekammer ist aus der im Jahre 1947 gegründeten Arbeitsgemeinschaft der Westdeutschen Ärztekammern hervorgegangen. Heute ist sie die Arbeitsgemeinschaft der 17 Landesärztekammern und somit ein organisatorischer $\mathrm{Zu}$ sammenschluss von Körperschaften öffentlichen Rechts. Der einzelne Arzt gehört der Bundesärztekammer lediglich mittelbar über die Pflichtmitgliedschaft in seiner Ärztekammer an. Die Bundesärztekammer selbst ist keine Körperschaft des öffentlichen Rechts, sondern ein privatrechtlicher nicht eingetragener Verein [www.bundesaerztekammer.de/05/index.html (Abrufdatum: 25. November 2004)].

\section{Qualifikation der Richtlinien der Bundesärztekammer}

Nach allgemeiner Ansicht sind diese Richtlinien der Bundesärztekammer nicht als zwingendes Recht anzusehen. Es handelt sich vielmehr um gesetzlich basierte fachliche Empfehlungen, die wissenschaftliche Erkenntnisse flexibel fortschreiben sollen. Grundsätzlich können daher auch von diesen Empfehlungen abweichende Standards zur Anwendung kommen, soweit diese gut begründet und evaluiert sind [Von Auer: Das Transfusionsgesetz von 1998: Chance und Herausforderung für die Transfusionsmedizin. Transfus Med Hemother 2004;31(suppl 2):104-108, S. 105 ].

Unmittelbare standes- bzw. berufsrechtliche Verbindlichkeit für die Ärzteschaft erlangen diese Richtlinien erst durch Umwandlung in Recht der Landesärztekammern auf Grundlage der Kammer- und Heilberufsgesetze der Bundesländer [Von Auer, Seitz: Gesetz zur Regelung des Transfusionswesens, Kommentar und Vorschriftensammlung, Stand Mai 2003, § 12 TFG, Rn. 2]. Im Verhältnis zu Dritten, also z.B. zu Patienten, erhalten diese Richtlinien als Sorgfaltsgebote über $§ 276$ Bürgerliches Gesetzbuch (BGB) in der Fassung der Bekanntmachung vom 2. Januar 2002 [BGB1. I S. 42], zuletzt geändert durch das Gesetz zur Modernisierung des Kostenrechts vom 5. Mai 2004 [BGBl. I S. 718] Bedeutung [Von Auer, Seitz: a.a.O] Die Hämotherapierichtlinien wirken also unmittelbar nur in Bezug auf die Ärzteschaft, eine Wirkung für die Allgemeinheit (erga omnes) entfalten sie allenfalls mittelbar. Sie haben daher keine allgemeine Rechtsnormqualität.

\section{Erfüllung der EG-Vorgaben}

Nunmehr ist zu fragen, ob durch den Erlass dieser Richtlinien durch die Bundesärztekammer die Vorgaben der Arti- der Richtlinie 2004/33/EG eingehalten werden können. Diese Normen fordern den Erlass der zur Umsetzung notwendigen Rechts- und Verwaltungsvorschriften durch die Mitgliedsstaaten.

Der gemeinschaftsrechtliche Begriff der Rechts- und Verwaltungsvorschrift ist weit zu verstehen. Grundsätzlich sind von diesem Terminus alle Gesetze, Verordnungen, Kollektivverträge, Verwaltungsverordnungen, das Gewohnheitsrecht, die ständige Rechtsprechung sowie die Verwaltungspraxis erfasst [Bleckmann, Europarecht, 6. Aufl. Köln, Haymanns, 1997, Rn. 2107].

\section{Gesetz}

Ein Gesetz im Sinne des deutschen Rechts [siehe hierzu im Einzelnen Wolff, Bachof, Stober: Verwaltungsrecht, Band 1, 11. Aufl. München, C.H. Beck, 1999, § 24, Rn. 7 ff] wird durch die Bundesärztekammer in keinem Fall erlassen. Grundsätzlich wird zwischen Gesetzen im formellen und Gesetzen im materiellen Sinn unterschieden. Ein Gesetz im formellen Sinne, das als ein in einem verfassungsmäßigem (förmlichen) - ordentlichen oder außerordentlichen - Gesetzgebungsverfahren zustande gekommener Willensakt der Gesetzgebungsorgane [Wolff, Bachof, Stober: a.a.O., § 24, Rn. 34] verstanden wird, liegt nicht vor. Weder kann von einem Willensakt der Gesetzgebungsorgane gesprochen werden, noch ist ein verfassungsmäßiges Gesetzgebungsverfahren gegeben. Auch ein Gesetz im materiellen Sinne wird durch die Bundesärztekammer nicht erlassen. Charakteristisch für ein allgemein bindendes Gesetz im materiellen Sinn ist der Erlass desselben durch die Legislative bzw. der Erlass einer Rechtsverordnung durch die Exekutive aufgrund einer Ermächtigung durch die Legislative und eine Anwendungsverpflichtung für die Gerichtsbarkeit [Wolff, Bachof, Stober: a.a.O., § 24, Rn. 13]. Gerade Letzteres ist aber nicht der Fall, da die Bundesärztekammer lediglich gesetzlich basierte fachliche Empfehlungen geben soll, die der Fortschreibung wissenschaftlicher Erkenntnisse dienen. Grundsätzlich besteht also keine gerichtliche Bindung, da auch von diesen Empfehlungen abweichende Standards zur Anwendung kommen können, soweit diese gut begründet und evaluiert sind [Von Auer: a.a.O., S. 105].

\section{Rechtsverordnung}

Gleichfalls nicht in Betracht kommt der Erlass einer Rechtsverordnung durch die Bundesärztekammer. Rechtsverordnungen sind materielle Gesetze, die auf der Grundlage einer nach Inhalt, Zweck und Ausmaß begrenzten gesetzlichen Ermächtigungsnorm (Art. 80 Abs. 1 S. 2 GG) erlassen wurden und in bestimmter Form hoheitlich einseitig erlassene, abstrakte und meist generelle Anordnungen von exekutiven 
Regierungs- und Verwaltungsorganen zur Regelung des allgemeinen staatlichen Gewaltverhältnisses mit materiellem Gesetzescharakter sind [Wolff, Bachof, Stober: a.a.O., § 25, Rn. 30]. Zum einen ist die Bundesärztekammer kein Regierungs- oder Verwaltungsorgan, sondern ein privatrechtlich organisierter nichtrechtsfähiger Verein, zum anderen fehlt den Richtlinien der Bundesärztekammer - wie bereits dargestellt - der Charakter eines materiellen Gesetzes.

\section{Kollektivvertrag}

Auch die Annahme eines Kollektivvertrages erscheint abwegig. Zivilrechtlich betrachtet ist ein Vertrag die von zwei oder mehr Personen erklärte Willensübereinstimmung über die Herbeiführung eines bestimmten rechtlichen Erfolgs [Palandt, Bürgerliches Gesetzbuch, 62. Aufl. München, Beck Juristischer Verlag, 2003, Einf. v. § 145, Rn. 1]. Im Bereich des öffentlichen Rechts wird der Begriff des öffentlich-rechtlichen Vertrags durch § 54 Verwaltungsverfahrensgesetz (VwVfG) in der Fassung der Bekanntmachung vom 23. Januar 2003 [BGBl. I S. 102], zuletzt geändert durch das Gesetz zur Modernisierung des Kostenrechts vom 5. Mai 2004 [BGB1. I S. 718], legal definiert. Demnach kann ein Rechtsverhältnis auf dem Gebiet des öffentlichen Rechts durch Vertrag begründet, geändert oder aufgehoben werden, soweit dem Rechtsvorschriften nicht entgegenstehen.

Trotz des von $\S \S 12$ Absatz 1 und 18 Absatz 1 TFG geforderten Einvernehmens zwischen Bundesärztekammer und der zuständiges Bundesoberbehörde kann nicht vom Entstehen einer irgendwie gearteten Vertragsbeziehung ausgegangen werden. Dagegen spricht der Wortlaut des TFG, der von einer bloßen deklaratorischen Feststellung des Standes von Wissenschaft und Technik durch die Bundesärztekammer ausgeht, also einen einseitigen Vorgang im Auge hat. Außerdem ist das Einvernehmen zwischen Bundesärztekammer und zuständiger Bundesoberbehörde nicht auf einen zwischen beiden wirkenden rechtlichen Erfolg, sondern auf den Erlass bestimmter Richtlinien gerichtet.

\section{Verwaltungsvorschriften}

Die Richtlinien der Bundesärztekammer sind auch nicht als Verwaltungsvorschriften einzuordnen. Nach der deutschen verwaltungsrechtlichen Dogmatik werden Verwaltungsvorschriften als hoheitliche Rechtssätze eines leitenden Organs oder Amtes definiert, die sich unmittelbar lediglich an ihre weisungsabhängigen Glieder, Organe und Ämter als Institutionen richten und die Wahrnehmung ihrer amtlichen, durch Rechtssatz bestimmten Zuständigkeiten kraft Weisungsgewalt des Näheren regeln [Wolff, Bachof, Stober: a.a.O., § 24, Rn. 22]. Die Bundesärztekammer als nichtrechtsfähiger Verein und Zusammenschluss der Landesärz- tekammern steht zu diesen nicht in einem Über-/Unterordnungsverhältnis und kann den Landesärztekammern keine Weisungen erteilen. Gleiches gilt für die Ärzteschaft. Der einzelne Arzt ist lediglich Mitglied in den für ihn zuständigen Landesärztekammern bzw. Ärztekammern. Da insoweit keine Mitgliedschaft in der Bundesärztekammer besteht, kann auch von einer Weisungsmöglichkeit der Bundesärztekammer an den einzelnen Arzt nicht die Rede sein. Es fehlt also bereits an der ersten charakteristischen Voraussetzung einer Verwaltungsvorschrift, nämlich der Weisungsbefugnis des erlassenden Organs gegenüber dem empfangenden Organ.

\section{Gewohnheitsrecht}

Die Richtlinien der Bundesärztekammer können auch nicht als Gewohnheitsrecht eingeordnet werden. Gewohnheitsrecht besteht dann, wenn ein bestimmtes Verhalten, ohne ausdrücklich hoheitlich geboten zu sein, von den Beteiligten in der Überzeugung rechtlicher Gebotenheit oder Gewährung so lange gleichmäßig und allgemein geübt worden ist, dass das Gleichheitsgebot einer Abweichung entgegensteht [Wolff, Bachof, Stober: a.a.O., § 25, Rn. 12]. Im vorliegenden Fall geht es um die Umsetzung neuer Standards für die Gewinnung und Anwendung von Blut bzw. Blutprodukten. Das Vorliegen von Gewohnheitsrecht ist daher schon begrifflich ausgeschlossen.

\section{Ständige Rechtsprechung}

Die Fallgruppe der ständigen Rechtsprechung ist ausgeschlossen, da keine Handlungen staatlicher Gerichte vorliegen.

\section{Verwaltungspraxis}

Auch eine Verwaltungspraxis ist in den Richtlinien nicht $\mathrm{zu}$ sehen, da diese ausweislich des Wortlauts der $\S 12$ Absatz 1 und 18 Absatz 1 TFG von der Bundesärztekammer, also einem nichtrechtsfähigem Verein, und nicht von einer Stelle der öffentlichen Verwaltung erlassen werden.

Durch die geplante Umsetzung der Richtlinien 2002/98/EG und 2004/33/EG in den Hämotherapierichtlinien der Bundesärztekammer werden die Vorgaben der Artikel 32 Absatz 1 der Richtlinie 2002/98/EG und 9 Absatz 1 der Richtlinie 2004/33/EG nicht erfüllt. Die Richtlinien der Bundesärztekammer sind nicht als Rechts- und Verwaltungsvorschriften im gemeinschaftsrechtlichen Sinne zu qualifizieren. Maßgeblich ist für diese Einschätzung vor allem, dass in den Handlungen der Bundesärztekammer kein hoheitliches Handeln gesehen werden kann. Nichts anderes ergibt sich dadurch, dass eine Veröffentlichung im Bundesanzeiger geplant ist. Die Ver- 
öffentlichung ist - wie sich aus der obigen Darstellung ergibt kein konstitutives Erfordernis für die Qualifikation als Rechts- und Verwaltungsvorschriften im gemeinschaftsrechtlichen Sinn.

\section{Weitere Überlegungen}

\section{Beleihung der Bundesärztekammer}

Etwas anderes könnte sich ergeben, wenn man zu dem Ergebnis käme, die Bundesärztekammer sei durch die Regelung der $\S \S 12$ Absatz 1, 18 Absatz 1 TFG vom Gesetzgeber mit öffentlich-rechtlichen, nämlich legislativen Befugnissen beliehen worden. Dann wäre das Handeln der Bundesärztekammer als hoheitlich zu qualifizieren, was zu einer anderen Betrachtung führen könnte.

Beliehene sind Private (Einzelpersonen oder juristische Personen des Privatrechts), die mit der Wahrnehmung bestimmter Verwaltungsaufgaben im eigenen Namen betraut sind [Maurer: Allgemeines Verwaltungsrecht, 13. Aufl. München, C.H. Beck, 2000, § 23 Rn. 56]. Problematisch ist es aber insoweit, dass es sich bei der Bundesärztekammer um einen nicht eingetragenen Verein handelt. Da ein Idealverein (um einen solchen dürfte es sich bei der Bundesärztekammer handeln, da ihr Zweck nicht auf einen wirtschaftlichen Geschäftsbetrieb gerichtet ist) nach $\S 21$ BGB Rechtsfähigkeit durch Eintragung in das Vereinsregister erlangt, fehlt es der Bundesärztekammer an der für eine Beleihung durch den Gesetzgeber notwendigen eigenen Rechtspersönlichkeit.

Dementsprechend werden die Regelungen in $\S \S 12$ Absatz 1 und 18 Absatz 1 TFG auch als «Auftrag» des Gesetzgebers bezeichnet [Von Auer, Seitz: a.a.O., § 12 TFG, Rn. 4]. Zwar entsteht durch eine Beleihung auch ein öffentlich-rechtliches Auftragsverhältnis zwischen Hoheitsträger und Beliehenem [Maurer: a.a.O., § 23, Rn. 58], die Bundesärztekammer hat aber als nicht eingetragener Verein keine Möglichkeit, Verwaltungsakte zu erlassen [Von Auer, Seitz: a.a.O., § 12 TFG, Rn. 4] und unterliegt auch nicht der Aufsicht durch den Bund.

Eine Beleihung der Bundesärztekammer durch die $\S \S 12$ Absatz 1, 18 Absatz 1 TFG kommt demnach nicht in Betracht. Für den Erlass der bisherigen Richtlinien wurde in der Literatur 1998 noch festgestellt, dass durch die $\S \S 12,18$ TFG «keine Übertragung gesetzgeberischer Aufgaben an Außenstehende» erfolgt sei [Deutsch: Sicherheit bei Blut und Blutprodukten: Das Transfusionsgesetz von 1998. NJW, 1998, S. 3377-3381, S. 3379 ]. Diese Feststellung kann für die beabsichtigte Umsetzung von EG-Richtlinien durch die Bundesärztekammer nicht mehr aufrecht erhalten werden. Im Jahre 2003 hat Taupitz [Richtlinien in der Transplantationsmedizin, Neue Jur Wochenschr 2003;56:1145-1150] für den Bereich der Transplantationsmedizin - Analoges gilt für den Blutbereich - zutreffend festgestellt, dass die Bundesärztekammer keine Rechtsset- zungsgewalt besitzt. Diese sei ihr weder durch die Kammern noch durch Gesetz übertragen worden. Die Richtlinien der Bundesärztekammer seien als solche kein Recht, allenfalls handele es sich um «normkonkretisierende Verweisungen». Sie haben den «Charakter von antizipierten Sachverständigengutachten» zum jeweils in ihnen festgehaltenen Stand der medizinischen Wissenschaft. Eine solche rechtliche Qualifikation reicht jedoch für die rechtlich zwingende Umsetzung von EG-Richtlinieninhalten nicht aus.

\section{Beteiligung der zuständigen Bundesoberbehörde}

Die Tatsache der Beteiligung der zuständigen Bundesoberbehörde (Einvernehmen) beim Erlass der Hämotherapierichtlinien ändert nichts an der Tatsache, dass diese Richtlinien durch ein nichtstaatliches Organ erlassen werden. Der Wortlaut der Normen geht eindeutig von einer Feststellung durch die Bundesärztekammer und nicht durch das Paul-EhrlichInstitut als nach $\S 27$ Absatz 1 TFG zuständiger Bundesoberbehörde aus.

\section{EG-rechtliche Erwägungen}

Durch das im deutschen Transfusionsrecht geplante Verfahren wird die in den EG-Richtlinien vorgeschriebene Form der Umsetzung durch Rechts- und Verwaltungsvorschriften nicht beachtet. Insoweit liegt nicht nur ein Verstoß gegen die Umsetzungsgebote der Richtlinien 2002/98/EG bzw. 2004/33/EG vor, sondern auch gegen Artikel 10 EGV.

Möglicherweise könnte sich jedoch aus der Rechtsprechung des Europäischen Gerichtshofs (EuGH) zur Richtlinienumsetzung eine andere Sichtweise ergeben. Grundsätzlich müssen die Richtlinien den Mitgliedsstaaten nämlich die Wahl der konkreten Form der Umsetzung offen lassen (vergleiche Art. 249 Abs. 3 EG-Vertrag). Hintergrund dieser Verpflichtung ist, dass die einzelnen Staaten ihre Gesetzgebungsbefugnisse grundsätzlich behalten haben, folglich also aus eigenem Recht handeln, und nicht aufgrund einer Delegation oder Ermächtigung des europäischen Rechts [Bleckmann: a.a.O., Rn. 441].

Trotz dieser grundsätzlichen Freiheit der Mitgliedsstaaten existiert umfangreiche Rechtsprechung des EuGH zu der Frage, wie Richtlinien der Europäischen Gemeinschaft in nationales Recht umzusetzen sind. Hierbei hat der EuGH zu verschiedenen Einzelfragen dieses Themenkomplexes Stellung genommen. Soweit ersichtlich war der EuGH aber noch nicht mit der hier relevanten Frage konfrontiert, dass die Umsetzung maßgeblich durch einen nichtrechtsfähigen Verein erfolgen soll. Insoweit ist zu prüfen, ob das hier geplante Verfahren mit den bisher existierenden Vorgaben vereinbar ist. 


\section{Allgemeine Vorgaben}

Grundsätzlich sind die in der Richtlinie festgelegten Verpflichtungen und Fristen von den Mitgliedstaaten zu beachten. Insbesondere ist die Berufung eines Mitgliedsstaates auf entsprechende Bestimmungen, Übungen oder Umstände seiner internen Rechtsordnung nicht geeignet, eine Nichtumsetzung zu rechtfertigen [Weber: Zur Umsetzung von EG-Richtlinien im Umweltrecht. Umwelt- und Planungsrecht 1992:5-9, S. 6 mit weiteren Nachweisen (m.w.N.)].Dieser Fall liegt nicht vor, da die Richtlinie umgesetzt werden soll und keine Berufung darauf erfolgt, in der Bundesrepublik Deutschland bestünde bereits ein den Vorgaben der Richtlinie entsprechender Rechtszustand.

Außerdem gesteht der EuGH dem Bürger das Recht zu, sich vor den nationalen Gerichten der Mitgliedsstaaten darauf zu berufen, eine mit einer von dem betreffenden Mitgliedsstaat nicht rechtzeitig umgesetzten Richtlinie unvereinbare Norm dürfe ihm gegenüber nicht angewandt werden [Weber: a.a.O., S. 6, m.w.N.). Auch dieser Problemkreis spielt hier keine Rolle, da es nicht um das «Ob», sondern um das «Wie» einer Umsetzung geht.

\section{Vorgaben zur Qualität des Umsetzungsaktes}

In Bezug auf die Qualität der innerstaatlichen Umsetzungsakte hat der EuGH zunächst gefordert, dass die Mitgliedsstaaten diejenigen Formen und Mittel zu wählen haben, die für die Gewährleistung der praktischen Wirksamkeit (effet utile) am besten geeignet sind [Weber: a.a.O., S. 6, unter Bezugnahme auf EuGH Slg. 1976, 517]. Später wurde dann grundlegend festgestellt, dass Richtlinien in einzelstaatliche Vorschriften umgesetzt werden müssen, denen dieselbe Qualität zukommt wie jenen, die in diesem Mitgliedsstaat generell für die zu regelnde Materie gelten [Weber: a.a.O., S. 6, unter Bezugnahme auf EuGH Slg. 1980, 1473 ff (1486)].

Betrachtet man diese beiden Erfordernisse für sich, so ist die in Deutschland geplante Umsetzung der Richtlinien 2002/98/EG und 2004/33/EG durch Richtlinien der Bundesärztekammer mit den Vorgaben des EuGH unvereinbar. Zwar gelten in der Bundesrepublik Deutschland für die zu regelnde Materie generell bisher die lediglich standesrechtlich verbindlichen Hämotherapierichtlinien der Bundesärztekammer. Die praktische Wirksamkeit der Vorgaben (effet utile) der Richtlinien jedoch ist durch die geplante Umsetzung seitens der Bundesärztekammer aus zwei Gründen nicht ausnahmslos gewährleistet.

Zum einen handelt es sich bei den Richtlinien der Bundesärztekammer um gesetzlich basierte fachliche Empfehlungen, die wissenschaftliche Erkenntnisse flexibel fortschreiben sollen und grundsätzlich auch erlauben, dass von diesen Empfehlungen abweichende Standards zur Anwendung kommen können, soweit diese gut begründet und evaluiert sind [Von Auer: a.a.O., S. 105].
Zum anderen besteht zwischen den Staats- und Verwaltungsorganen der Bundesrepublik Deutschland und der Bundesärztekammer kein Subordinationsverhältnis. Damit kann die Bundesrepublik Deutschland nicht garantieren und verbindlich und zeitgerecht bewirken, dass das innerstaatliche Recht auf diesem Gebiet tatsächlich immer den europäischen Vorgaben entsprechen wird. Im Fall einer Meinungsverschiedenheit z.B. über den Inhalt, die Notwendigkeit oder den Zeitpunkt des Erlasses von zu ändernden Hämotherapierichtlinien könnte die Bundesärztekammer rechtlich nicht wie eine staatliche Behörde angewiesen werden, Richtlinien eines bestimmten Inhalts zu erlassen. Auch könnte die Bundesärztekammer ihre Richtlinie möglicherweise jederzeit ändern, wenngleich dies durch das erforderliche Einvernehmen des Paul-EhrlichInstituts im Ergebnis verhindert werden könnte.

Zwar ist nach der Rechtsprechung des EuGH zur Umsetzung einer Richtlinie nicht unbedingt und in jedem Fall ein Tätigwerden des Gesetzgebers erforderlich [Jarras, Beljin: Grundlagen des EG-Rechts. Baden-Baden, Nomos, 2003, S. 281]. Auch verfassungs- und verwaltungsrechtliche Grundsätze können ausreichen, wenn die sich aus diesen Grundsätzen ergebende Rechtslage, soweit die Richtlinie Ansprüche Einzelner begründen soll, hinreichend bestimmt und klar ist und die Begünstigten von ihren Rechten Kenntnis erlangen könnten [Weber: a.a.O., S. 6, unter Bezugnahme auf EuGH Slg. 1985, $1661 \mathrm{ff}$ (1673)]. Allerdings existieren im vorliegenden Fall keine verfassungs- oder verwaltungsrechtlichen Grundsätze, die ein Tätigwerden des Gesetzgebers entbehrlich erscheinen ließen. Die Richtlinien der Bundesärztekammer stellen zum einen, wie bereits dargelegt, keine verfassungs- oder verwaltungsrechtlichen Grundsätze dar, und zum anderen entsprechen sie rechtlich noch nicht dem Rechtsstandard, der durch die Umsetzung der Richtlinie europaweit erreicht werden soll. Die Urteile vom 30. Mai 1991 [EuGH Slg. 1991, 2567 ff und $2607 \mathrm{ff}]$ haben die Rechtsprechung des EuGH auf diesem Gebiet weiter entwickelt. Vor diesen Urteilen betrachtete der EuGH einer Richtlinienumsetzung als ungenügend, wenn die vom Mitgliedsstaat gewählte Form der Umsetzung eine beliebige Abänderbarkeit der innerstaatlichen Rechtslage und mangelnde Publizität der Umsetzung mit sich brachte (am Beispiel der Richtlinienumsetzung durch Verwaltungspraxis). Hintergrund dieser Rechtsprechung war die Forderung nach Rechtssicherheit für die Gemeinschaftsbürger [Von Danwitz: Normenkonkretisierende Verwaltungsvorschriften und Gemeinschaftsrecht. Verwaltungsarchiv 1993;84:73-96, S. 80]. In beiden Urteilen begründete der EuGH das Erfordernis der Richtlinienumsetzung durch Rechtssatz mit der den Betroffenen von den entsprechenden Richtlinien eingeräumten subjektiven Rechtsstellung [Von Danwitz, a.a.O., S. 80]. Damit besteht nach der Rechtsprechung des EuGH ein Rechtssatzvorbehalt kraft Gemeinschaftsrechts jedenfalls dann, wenn die umzusetzenden Richtlinien subjektive Rechte Einzelner begründen oder ordnungsrechtliche Maßnahmen ermöglichen sollen [Von 
Danwitz, a.a.O., S. 81]. Nach Artikel 1 der Richtlinie 2002/98/ EG besteht ihr Ziel darin, ein hohes Niveau des Gesundheitsschutzes zu gewährleisten. Da die Richtlinie 2004/33/EG der Durchführung der Richtlinie 2002/98/EG dient, muss sie dasselbe Anliegen haben. Damit begründen die Vorgaben der Richtlinien ein subjektives Recht des Bürgers, einen den Richtlinien entsprechenden Standard sowohl z.B. als Spender (Gewinnung von Blut), als auch z.B. als Patient (Anwendung von Blutprodukten) vorzufinden. Geht man von dieser Vorgabe aus, so ist nach der Rechtsprechung des EuGH eine Umsetzung durch Rechtssatz erforderlich

Die geplante Art der Umsetzung erfüllt damit nicht die Vorgaben der Rechtsprechung des EuGH, da weder die praktische Wirksamkeit der Vorgaben in jedem Fall gewährleistet ist noch eine Umsetzung - wie von der Rechtsprechung des EuGH für den Fall der Einräumung subjektiver Rechte durch die Richtlinie gefordert - durch Rechtssatz geplant ist.

\section{Deutsches Verfassungsrecht}

Auch unter verfassungsrechtlichen Gesichtspunkten kann die vorgesehene Erweiterung der Kompetenzen der Bundesärztekammer Bedenken hervorrufen. Bisher wurde der Stand der medizinischen Wissenschaft und Technik durch die Bundesärztekammer festgestellt, was aufgrund der Kompetenz und Sachnähe der Ärzteschaft unter rechtlichen und Selbstverwaltungsgesichtspunkten vertretbar erscheint. Zukünftig sollen jedoch auch europarechtlich festgelegte Mindeststandards durch die Bundesärztekammer auf nationaler Ebene «umgesetzt» werden. Dies ist eine klassische Aufgabe des Gesetzgebers. Daher ist nicht auszuschließen, dass hierdurch der verfassungsrechtliche Grundsatz des Parlamentsvorbehalts und die Wesentlichkeitslehre verletzt werden.

Der Parlamentsvorbehalt betrifft die Frage, welche Regelungen durch die Legislative selbst getroffen werden müssen und welche von ihr delegiert werden können [Lücke in Sachs (Hrsg): Grundgesetz, Kommentar München, C.H. Beck, 1996, Art. 80, Rn. 19]. Grundsätzlich gilt hierbei, dass Angelegenheiten von besonderer Wichtigkeit, die etwa miteinander kollidierende Grundrechte in Einklang bringen sollen, durch Parlamentsgesetz zu regeln sind. Erst wenn diese besondere Wichtigkeit nicht gegeben ist, soll eine Regelung der Materie aufgrund einer Ermächtigung des parlamentarischen Gesetzgebers möglich sein [Lücke in Sachs, a.a.O.]. Nach der Konzeption des Gesetzes soll der Bundesärztekammer eine klassische Aufgabe des Gesetzgebers, nämlich die Umsetzung von EG-Richtlinien, abstrakt übertragen werden. Dies kann unter dem Gesichtspunkt des Parlamentsvorbehalts dann zu verfassungsrechtlichen Problemen führen, wenn einer der zukünftig umzusetzenden EG-Richtlinien zur Beeinträchtigung von Grundrechten einer von den Hämotherapierichtlinien betroffenen Person führt.

Gleiches gilt für die Wesentlichkeitslehre, die davon ausgeht, der Gesetzgeber sei verpflichtet, in grundlegenden normativen Bereichen (zu denen jedenfalls der Bereich der Grundrechtsausübung gezählt wird), alle wesentlichen Entscheidungen selbst zu treffen [Stober, Wolff, Bachof: a.a.O., § 18, Rn. 12]. Auch hier mag die bisherige Rechtslage aufgrund der Sachnähe der Ärzteschaft gerechtfertigt sein, die abstrakte nunmehr beabsichtigte Übertragung der EG-Richtlinienumsetzung durch die Bundesärztekammer führt aber spätestens dann zu Schwierigkeiten, wenn eine umzusetzende EG-Richtlinie Grundrechtsbeeinträchtigungen beinhaltet.

\section{Richtlinienumsetzung auf dem Gebiet des Arzneimittelrechts}

Im Arzneimittelrecht sieht $\S 83$ Absatz 1 des Gesetzes über den Verkehr mit Arzneimitteln (Arzneimittelgesetz; AMG) in der Fassung der Bekanntmachung vom 11. Dezember 1998 [BGBl. I S. 3586], zuletzt geändert durch das Zwölfte Gesetz zur Änderung des Arzneimittelgesetzes vom 30. Juli 2004 [BGB1. I S. 2031], den Erlass von Rechtsverordnungen oder allgemeinen Verwaltungsvorschriften zum Zwecke der Angleichung der Rechts- und Verwaltungsvorschriften der Mitgliedsstaaten der Europäischen Wirtschaftsgemeinschaft vor, soweit dies zur Durchführung von Verordnungen, Richtlinien oder Entscheidungen des Rates oder Europäischen Kommission der Europäischen Gemeinschaften erforderlich ist. Die Vorschrift räumt dem Verordnungsgeber die Möglichkeit ein, im Rahmen des bestehenden Ermächtigungssystems die Anpassung an verbindlich gewordene EG-Richtlinien zu vollziehen [Kloesel, Cyran, Feiden, Pabel (Hrsg): Arzneimittelrecht - Kommentar 3. Auflage, Stand: 2004. Stuttgart, Wissenschaftliche Verlagsanstalt, 2004, § 83 AMG (Amtliche Begründung)]. Darüber hinaus hat es sich als notwendig erwiesen, die Anpassung an Gemeinschaftsrecht auch durch allgemeine Verwaltungsvorschriften zu vollziehen (Kloesel, Cyran, Feiden, Pabel: a.a.O. (4. Änderungsgesetz Amtliche Begründung)].

Aus dieser Norm ist der Schluss zu ziehen, dass der Gesetzgeber auf dem Gebiet des Arzneimittelrechts davon ausgeht, dass eine Umsetzung von EG-Richtlinien durch Vorschriften, die in der Normenhierarchie unterhalb von Verwaltungsvorschriften anzusiedeln sind, ausgeschlossen ist. Auf dem Gebiet des TFG kann nichts anderes gelten.

Darüber hinaus sieht ein Gesetzentwurf der Bundesregierung zur Änderung arzneimittelrechtlicher Vorschriften [BR-Drs. $875 / 04$ vom 5. November 2004] vor, dass die nach § 26 AMG bisher in der Form allgemeiner Verwaltungsvorschriften erlassenen Arzneimittelprüfrichtlinien künftig als Rechtsverordnung erlassen werden sollen. Als Begründung für diese Änderung wird angeführt, dass die Richtlinien der EG, welche die Anforderungen an Zulassungs- und Registrierungsverfahren (RL 2001/83/EG und RL 2001/82/EG) vorgeben, so beschaffen seien, dass sich ihre Umsetzung in nationales Recht nicht 
auf Anweisungen an die Zulassungsbehörde zur Prüfung der eingereichten Unterlagen beschränken lasse, sondern Vorschriften erfordere, die sich - als Rechtsnormen - unmittelbar an den Antragsteller richten. Daher solle nunmehr in $\S 26$ AMG eine Verordnungsermächtigung geschaffen und die Arzneimittelprüfrichtlinien als Rechtsverordnung erlassen werden. Auch hierbei handelt es sich um die Umsetzung von EG-Richtlinienrecht, nämlich des Anhangs I der Richtlinie 2001/83/EG zur Schaffung eines Gemeinschaftskodexes für Humanarzneimittel.

Diese Richtlinie 2001/83/EG des Europäischen Parlaments und des Rates vom 6. November 2001 zur Schaffung eines Gemeinschaftskodexes für Humanarzneimittel (ABl. EU Nr. L 311/67 vom 28. November 2001) und die Richtlinie 2001/82/EG des Europäischen Parlaments und des Rates vom 6. November 2001 zur Schaffung eines Gemeinschaftskodex für Tierarzneimittel (AB1. EU Nr. L 311/1 vom 28. November 2001) verfolgen das Ziel des Schutzes der öffentlichen Gesundheit (vergleiche Erwägungsgrund 2 der Richtlinien). Genau dasselbe Ziel verfolgen, wie bereits dargestellt, aber auch die Blutrichtlinien 2001/98/EG und 2004/33/EG. Warum angesichts der identischen Zielrichtung der Richtlinien auf dem Gebiet des TFG ein im Vergleich zum Arzneimittelrecht entgegen gesetzter Umsetzungsweg beschritten wird, ist unverständlich und spricht vor dem Hintergrund der dargestellten Begründung des Gesetzentwurfs zur Änderung arzneimittelrechtlicher Vorschriften für die rechtliche Unzulässigkeit der vorgesehenen Umsetzungsform.

\section{Ergebnis}

Die geplante Art der Umsetzung der Blut-Richtlinien 2002/ 98/EG und 2004/33/EG durch Änderung der von der Bundesärztekammer nach $\S \S 12$ Absatz 1 und 18 Absatz 1 TFG bisher als bloßes Standesrecht erlassenen Hämotherapierichtlinien erfüllt weder die Vorgaben, welche die EG-Richtlinien selbst in Bezug auf ihre Umsetzung treffen, noch stimmt sie mit der Rechtsprechung des EuGH zur Qualität der Umsetzungsakte von Richtlinien überein. Auch in Bezug auf deutsches Verfassungsrecht bestehen angesichts der abstrakten
Übertragung der Umsetzungskompetenz für EG-Richtlinien auf die Bundesärztekammer erhebliche Zweifel an der Rechtmäßigkeit dieser Gesetzgebung.

Hieran ändert auch die geplante Veröffentlichung der Richtlinien im Bundesanzeiger nichts. Im konkreten Fall soll sie, wie bereits in der oben zitierten Gesetzesbegründung anklingt, den Hämotherapierichtlinien einen offiziellen Charakter verleihen. Die Veröffentlichung im Bundesanzeiger dient damit als Deckmantel, der den Anschein staatlicher Legitimation verleihen soll. Einen Erfahrungssatz dergestalt, dass eine äußere amtliche Form eines Dokuments konstitutiv für einen ebenfalls amtlichen Inhalt desselben spricht, gibt es nicht. Er wäre rechtlich völlig irreführend.

Trotz der gewünschten Flexibilität sind die Hämotherapierichtlinien der Bundesärztekammer weder der geeignete Ort noch das geeignete Instrument für die Umsetzung fachlicher Standards, die in europäischen Richtlinien vorgegeben werden. Vielmehr wäre es eine Umsetzung am Gesetzgeber vorbei und ohne dessen unmittelbare Kontrolle. Die Bundesrepublik Deutschland setzt damit voraussichtlich einen erheblichen Teil der Richtlinien 2002/98/EG und 2004/33/EG nicht ordnungsgemäß um.

Damit läge eine Verletzung der Umsetzungspflicht aus Artikeln 249 und 10 EGV vor, welche die Einleitung eines Vertragsverletzungsverfahrens der Europäischen Kommission gegen den Mitgliedsstaat Bundesrepublik Deutschland nach Artikel 226 ff EGV rechtfertigen könnte.

Ein nach den Vorgaben des europäischen und deutschen Rechts gangbarer und angesichts der dargestellten Probleme eindeutig vorzuziehender Weg wäre die Schaffung einer Ermächtigungsnorm im TFG zum Erlass einer Rechtsverordnung durch das Bundesministerium für Gesundheit und soziale Sicherung. Eine solche Rechtsverordnung würde an die Stelle der bisher lediglich standesrechtlich zu qualifizierenden Hämotherapierichtlinien der Bundesärztekammer treten. Damit wäre sowohl die allgemeine rechtliche Verbindlichkeit der umzusetzenden Regelungen als auch ein aus europarechtlicher Sicht ordnungsgemäßer Umsetzungsakt gewährleistet. Die Zeit einer lediglich standesrechtlichen Regelung wesentlicher Teile des Transfusionsrechts ist vorbei. 